\title{
Pulmonary artery sarcoma diagnosed using intravascular ultrasound images
}

\author{
Yoshiaki Okano, Toru Satoh, Teiji Tatewaki, Takeyoshi Kunieda, Satoru Fukuyama, \\ Naoki Miyazaki, Yasuo Beppu
}

Division of Cardiology

and Pulmonary

Vascular Medicine,

Department of

Internal Medicine,

National

Cardiovascular Center,

5-7-1 Fujishirodai,

Suita, Osaka, Japan

Y Okano

T Satoh

T Tatewaki

T Kunieda

Division of

Respiratology, Nippon

Steel Cooperation

Yawata Works

Hospital, 1-1-1

Haruno-cho,

Yahata-Higashi-ku,

Kitakyushu, Japan

$S$ Fukuyama

$\mathrm{N}$ Miyazaki

Division of

Orthopedics, National

Cancer Institute, 5-1-1

Tsukiji, Chuo-ku,

Tokyo, Japan

Y Beppu

Correspondence to:

Dr Y Okano, Director,

Division of Cardiology,

Shinshiro City Hospital, 32-1

Kitabata, Shinshiro, Aichi

441-13, Japan

Received 3 March 1997 Returned to author

30 May 1997

Revised version received

22 December 1997

Accepted for publication

13 January 1998

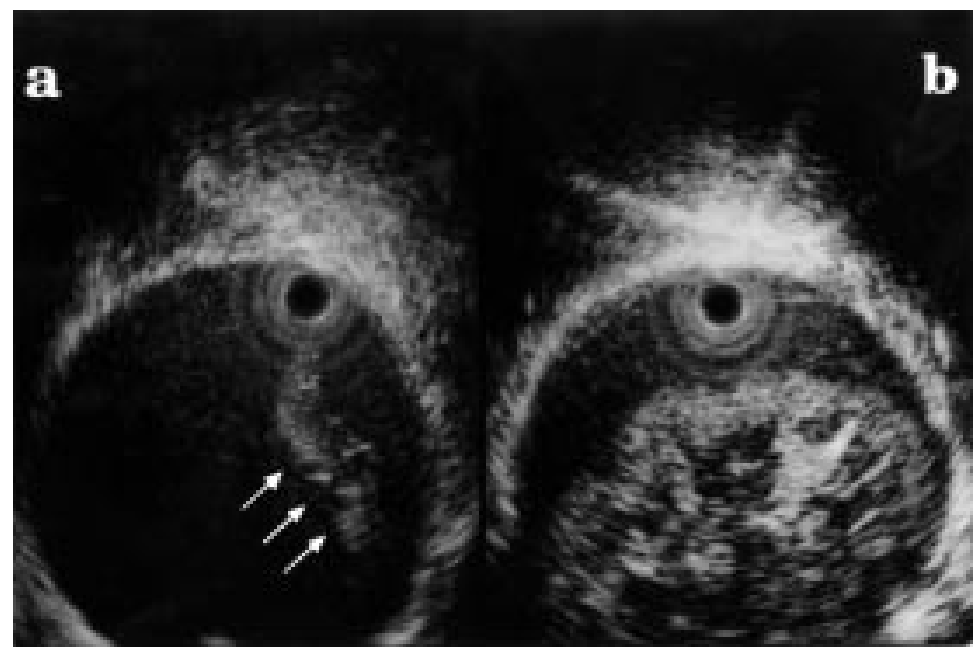

Figure 1 (a) A string shaped projection (arrows) was visualised in the proximal portion of the left main pulmonary artery by IVUS. (b) A unique echogenic mass was also visualised by IVUS which was continuous with the projection shown in (a) and had an irregular surface and a mosaic pattern containing flecked echolucent and increased echogenic areas. It was quite different from both echolucent fresh and echogenic organised thrombi.

\begin{abstract}
Primary and secondary malignant intravascular tumours of the pulmonary artery occur infrequently and the diagnosis is usually delayed as symptoms and findings from conventional examinations are nonspecific. The case is presented of a patient with a pulmonary artery sarcoma, probably arising from ribs resected some years previously, in which intravascular ultrasound (IVUS) provided important diagnostic findings.

(Thorax 1999;54:748-749)
\end{abstract} intravascular sarcoma

Although malignant tumours of the pulmonary arteries are rare ${ }^{1}$ they must be considered in the differential diagnosis of pulmonary embolism. Since the clinical presentation and conventional imaging features of such lesions are usually non-specific and mimic those of chronic thromboembolism, ${ }^{2}$ a definite diagnosis is often delayed until surgical exploration or necropsy. ${ }^{3}$ The following unusual case of pulmonary artery sarcoma is presented because of its interesting and diagnostic images obtained by intravascular ultrasound (IVUS).

\section{Case report}

A 66 year old man was referred to our hospital with the presumptive diagnosis of chronic thromboembolic pulmonary hypertension for
Keywords: pulmonary artery; intravascular ultrasound;

\section{(1)}

$$
5
$$
further clarification using a commercially available imaging system (SSD-550, Aloka Inc, Tokyo, Japan). A unique echogenic mass with an irregular surface, a string shaped projection (fig 1a), and a mosaic pattern containing flecked echolucent and increased echogenic areas (fig 1b) were clearly visualised by IVUS. These appearances differed from those seen in thromboembolic lesions ${ }^{4}$ and were diagnostic for a malignant pulmonary artery tumour. A second examination, including a biopsy specimen to confirm the histopathological diagnosis, was proposed but the patient declined. Attempts to locate the primary tumour and further metastases were unsuccessful. We scheduled surgical resection of the entire left lung. His respiratory condition, however, rapidly deteriorated and he died before the procedure. Postmortem necropsy revealed that the tumour of the left main pulmonary artery had 
grown into the vascular lumen, distally over the bifurcation of the left pulmonary artery. Histopathological examination of the tumour resulted in a diagnosis of a myxomatous chondrosarcoma showing a close resemblance to those in the ribs resected previously. We made a final diagnosis of secondary pulmonary artery chondrosarcoma by intravascular growth or embolisation $^{5}$ in this case, although we could not completely exclude the possibility of primary tumour.

\section{Discussion}

In this case IVUS allowed the diagnosis of malignant tumour in the pulmonary artery to be made prior to planned surgical intervention. Tumour involvements of the pulmonary vessels are not uncommon in necropsy cases of malignancy. ${ }^{6}$ However, most of these are microemboli and are infrequently detected clinically. Shepard et al reported only four cases of pulmonary artery intravascular tumour detected among 14000 consecutive cases after CT scanning of the chest and concluded that the findings of dilated and beaded peripheral pulmonary arteries on the CT scan are highly suggestive of intravascular tumour emboli. However, we found no such characteristic features on the CT scan in this case.

Gadolinium-enhanced MRI is an another potentially useful diagnostic tool to differentiate between intraluminal tumours and large thrombi. Although there is a case report in the literature documenting the value of gadoliniumenhanced MRI, ${ }^{8}$ our study did not show reliable differential diagnosis.

Ultrasound examinations seem to be more sensitive for qualitative evaluation of mass lesions. Only one case report, ${ }^{9}$ however, has been published, indicating the diagnostic significance of TTE in a patient with primary sarcoma of the pulmonary artery trunk. In many patients it is difficult to obtain a clear image even at the level of the pulmonary valve and pulmonary artery trunk using TTE. In addition, the left main portion of the pulmonary artery is difficult to visualise even though a transoesophageal approach is applied. In these circumstances IVUS presents a potential method for evaluating the intravascular lesions because of its ability to visualise directly from inside the vessel wall. ${ }^{10}$ Ricou et al $l^{4}$ suggested that IVUS might be useful in assessing the location and extension of the pathological process involving the pulmonary artery.

This work was supported in part by a grant from the Japan Cardiovascular Research Foundation. The authors are grateful to Kazuhiro Jinbo (Aloka, Osaka, Japan) for technical assistance in the IVUS procedure. We are also grateful to the following physicians and surgeons who participated in the medical care and the examination of the patient described in this report: Shingo Kyotani, Noritoshi Nagaya (Internal Medicine, National Cardiovascular Center), Motomi Ando, Shinichi Takamoto (Cardiovascular Surgery, National Cardiovascular Center), Tetsuro Naito (Naito Hospital, Tokyo, Japan).

1 Burke AP, Virmani R. Sarcomas of the great vessels. Cancer 1993;71:1761-73.

2 Delany SG, Doyle TCA, Bunton RW, et al. Pulmonary artery sarcoma mimicking pulmonary embolism. Chest 1993; 103:1631-3.

3 Baker PB, Goodwin RA Pulmonary artery sarcomas. A review and report of a case. Arch Pathol Lab Med 1985;109: 35-9.

4 Ricou F, Nicod P, Moser KM, et al. Catheter-based intravascular ultrasound imaging of chronic thrombo-
embolic pulmonary disease. Am f Cardiol 1991;67:749-52.

5 Schwarz MI, Goldman AL, Roycroft DW, et al. Vascular invasion by chondrosarcoma simulating pulmonary eminvasion by chondrosarcoma simulating
boli. Am Rev Respir Dis 1972;106:109-13.

6 Soares FA, Landell GA, de Oliveira JA. A prospective study Soares FA, Landell GA, de Oliveira JA. A prospective study
of the morphological aspects of tumor involvement of the of the morphological aspects of tumor invol
pulmonary vessels. Pathology 1992;24:150-4

7 Shepard JAO, Moore EH, Templeton PA, et al. Pulmonary intravascular tumor emboli: dilated and beaded peripheral pulmonary arteries at CT. Radiology 1993;187:797-801

8 Weinreb JC, Davis SD, Berkmen YM, et al. Pulmonary artery sarcoma: evaluation using Gd-DTPA. f Comput Assist Tomogr 1990;14:647-9.

9 Wright EC, Wellons HA, Martin RP. Primary pulmonary artery sarcoma diagnosed by two-dimensional echocardiography. Circulation 1983;67:459-62.

10 Pandian NG, Weintraub A, Kreis A, et al. Intracardiac, intravascular, two-dimensional, high frequency ultrasound imaging of pulmonary artery and its branches in humans and animals. Circulation 1990;81:2007-12. 